\title{
ORIGINAL ARTICLE Suicidal ideation in Korean persons with spinal cord injury
}

\author{
HS Nam ${ }^{1}$, HR Kim ${ }^{1,2}$, TH $\mathrm{Ha}^{3}$ and HI Shin ${ }^{1,2}$
}

Study design: Cross-sectional survey.

Objectives: To investigate the prevalence of suicidal ideation and attempt in Korean persons with spinal cord injury (SCI) and the factors related with suicidal ideation in comparison with a control group (CG).

Settings: Community-dwelling persons with chronic SCl in Korea.

Methods: A total of 382 persons with chronic SCl and 1104 able-bodied persons randomly selected from the general population and matched for age, sex and education level were compared. Suicidal ideation and attempt were recorded positive if the subjects had that experience during the past year. Psychosocial variables and injury-related factors were also evaluated.

Results: Suicidal ideation was found in 34.8\% (127/365) of SCI persons and $10.4 \%(115 / 1104)$ in the CG $(P<0.001)$. The rate of suicidal attempt among those with suicidal ideation was $17.3 \%(22 / 127)$ in $\mathrm{SCl}$ and $8.7 \%(10 / 115)$ in CG $(P=0.048)$. Multiple logistic regression revealed that SCI itself (odds ratio (OR) 2.865) was a significant risk factor of suicidal ideation. However, completeness of injury and injury level were not significantly associated with suicidal ideation. Depressive mood (OR 11.194, $P<0.001$ ) and female gender (OR 3.706, $P<0.001$ ) were significant risk factors of suicidal ideation in the $\mathrm{SCl}$ group with a predictive percentage of $77.5 \%$.

Conclusion: Suicidal ideation was more frequent among Korean persons with SCl compared with able-bodied peers and closely related to psychosocial adjustment. The results support the need for active intervention to prevent suicide regardless of injury severity. Spinal Cord (2013) 51, 789-793; doi:10.1038/sc.2013.92; published online 3 September 2013

Keywords: suicidal ideation; spinal cord injuries; risk factors; socioeconomic factors

\section{INTRODUCTION}

Suicidal risk is known to be greater in persons with spinal cord injury (SCI). ${ }^{1}$ DeVivo et al. ${ }^{2}$ reported that mortality due to suicide is 4.9 times higher in SCI persons compared with the general population. Hartkopp et al. ${ }^{3}$ also demonstrated that the total suicide rate among individuals with SCI was nearly five times greater than general population. Kishi et al. ${ }^{4}$ found that $7.3 \%$ of patients with acute medical illness and $11.7 \%$ of acute SCI persons had clinically significant suicidal ideation and that suicidal ideation occurred mostly among patients with depressive mood.

Psychosocial adjustment difficulties are known to be closely related to suicide in the SCI population. Hartkopp et al. ${ }^{3}$ reported that suicide rate in their group of marginally disabled persons with SCI was nearly twice as high as those with functionally complete tetraplegia. This suggests that psychosocial adjustment is related to degree of physical impairment.

The prevalence of suicidality and related factors can differ according to specific racial or cultural groups. For example, suicide is an urgent health issue in Korea with the rate of suicide increasing rapidly. ${ }^{5}$ However, data regarding suicidality of SCI persons in nonwestern cultures are still lacking.

This study was conducted to reveal the prevalence of suicidal ideation and attempt in community-dwelling SCI persons in Korea.
A further aim was to investigate the psychosocial and injury-related variables that could affect suicidal ideation, in comparison with an age, gender and educational level-matched non-SCI control group (CG) with consideration of the social setting and culture. We hypothesized that psychological factors would be closely related with suicidal ideation in SCI persons. To our knowledge, this is the first study to compare the rate of suicidal ideation in chronic SCI persons with the CG.

\section{MATERIALS AND METHODS}

\section{Subjects}

Subjects were members of the Korea Spinal Cord Injury Association (KSCIA). KSCIA is a non-government, non-profit organization (www.kscia.org). It is the only organization in Korea that was established to enhance the rights of SCI persons and is led by SCI persons themselves. Inclusion criteria were SCI persons aged $\geqslant 20$ years with post-injury duration of $>1$ year. A total of 382 subjects volunteered to participate in this survey. Fourteen subjects with incomplete data for education level were excluded from the analysis to perform CG matching. The resulting 368 subjects comprised the SCI group. The CG comprised of non-SCI subjects extracted from the database of 'The Fourth Korea National Health and Nutrition Examination Survey (KNHNES) 2009' performed on 10914 persons in the general population. KNHNES is a survey conducted by the Korean government every year on 20 randomly selected households in each of the 192 areas nationwide. The survey focuses on health

\footnotetext{
${ }^{1}$ Department of Rehabilitation Medicine, Seoul National University College of Medicine, Seoul, Republic of Korea; ${ }^{2}$ Department of Rehabilitation Medicine, Seoul National University Bundang Hospital, Gyeonggi-do, Republic of Korea and ${ }^{3}$ Department of Psychiatry, Seoul National University Bundang Hospital, Gyeonggi-do, Republic of Korea Correspondence: Dr HI Shin, Department of Rehabilitation Medicine, Seoul National University Bundang Hospital, 82, Gumi-ro 173 beon-gil, Bundang-gu, Seongnam, Gyeonggi-do 463-707, Republic of Korea.

E-mail: hyungik1@snu.ac.kr

Received 29 March 2013; revised 17 July 2013; accepted 24 July 2013; published online 3 September 2013
} 
state such as obesity, hypertension and diabetes mellitus, health behaviors such as drinking and smoking and also nutritional status. From this database, 1104 (1:3) subjects were chosen randomly, matched for age, gender and education level. We certify that all applicable institutional and governmental regulations concerning the ethical use of human volunteers were followed during the course of this research. This study was approved by the Institutional Review Board of Seoul National University Bundang Hospital (B-1109/135-010).

\section{Development of questionnaire}

A questionnaire based on the KNHNES 2009 was developed to investigate factors related to suicidal ideation among persons with SCI. Self-reported suicidal ideation and attempt were indicated if subjects had experienced these issues during the past year. Survey items reflecting demographic characteristics, social and economic status and physical and psychological conditions were selected from the KNHNES to secure comparability. This included gender, age and education level classified into three categories: elementary school or lower, middle to high school, and college or higher. Data relating to social and economic status, numbers of the family members living together, marital status, monthly income, home ownership and financial support (provided by the government or insurance) was also obtained. Physical and psychological conditions were assessed using a five-level rating scale for self-perceived health status (very good, good, normal, poor, very poor) and subjective body image (very skinny, skinny, normal, fat, very fat). Stress level encountered in daily life was assessed using a four-point scale (very low, low, high, very high). Characteristics of alcohol dependence was assessed by frequency of drinking, excessive drinking, uncontrolled drinking and drinking up to the point to affect their normal life on a five-level scale (none, less than once a month, once a month, once a week, almost every day). Abdominal obesity was measured by abdominal circumference, with threshold value of $90 \mathrm{~cm}$ in men and $85 \mathrm{~cm}$ in women. ${ }^{6}$ Depressive mood was defined as having feelings of sadness or despair affecting their daily lives in $\geqslant 2$ consecutive weeks during the past year. Additionally, age at the onset of SCI, motor completeness of the injury, injury level (tetraplegia or paraplegia) and duration since injury was included.

\section{Survey}

Following the development of the questionnaire, survey items were given to a convenience sample of $10 \mathrm{SCI}$ persons to ensure readability and comprehension of the questions. One investigator from each chapter of KSCIA, a total of 13 investigators, were recruited for the survey. Each investigator received $8 \mathrm{~h}$ of training before visiting the homes of participants in the SCI group.

\section{Statistical analysis}

Descriptive statistical analyses were performed to calculate the distribution of each factor in both the groups. The chi-square test was used to compare categorical data between the CG and the SCI group. The chi-square test was also used to test the independence between each factor and suicidal ideation, separately in both the CG and SCI groups. Multiple logistic regression for all the subjects, and also only for the SCI group, was performed to investigate significant factors related with suicidal ideation. All statistical analyses were carried out with SPSS 19.0 (SPSS Inc., Chicago, IL, USA).

\section{RESULTS}

Characteristics of the subjects in the CG and SCI group are shown in Table 1. In the SCI group, traumatic SCI consisted of $92.2 \%$ (333/ $361)$, compared with $7.8 \%$ (28/361) for non-traumatic injury. The proportion of motor complete (American Spinal Injury Association (ASIA) Impairment Scale (AIS) A, B) and motor incomplete injury (AIS C, D) was 61.9\% (223/360) and 38.1\% (137/360), respectively, and the proportion of tetraplegia and paraplegia was $38.3 \%(138 / 360)$ and $61.7 \%(222 / 360)$, respectively. Time since injury was $13.80 \pm 8.55$ years and the mean age at injury was $31.54 \pm 10.17$ years.

Prevalence of suicidal ideation in the SCI group was $34.8 \%(127 / 365)$ while it was $10.4 \%(115 / 1104)$ in the CG group $(P<0.001)$. Among
Table 1 Characteristics of subjects in CG and SCI

\begin{tabular}{|c|c|c|c|}
\hline & $C G$ & $\mathrm{SCl}$ & P-value \\
\hline Total number of cases & 1104 & 368 & \\
\hline Gender (male/female) & $915(82.9 \%) / 189$ & $305(82.9 \%) / 63$ & \\
\hline \multicolumn{4}{|l|}{ Age, years } \\
\hline 20-29 & $60 / 1104$ (5.4\%) & 20/368 (5.4\%) & \\
\hline 30-39 & 267/1104 (24.2\%) & $89368(24.2 \%)$ & \\
\hline $40-49$ & 435/1104 (39.4\%) & $145 / 368(39.4 \%)$ & \\
\hline $50-59$ & 270/1104 (24.5\%) & $90 / 368(24.5 \%)$ & \\
\hline$>60$ & $72 / 1104$ (6.5\%) & $24 / 368(6.5 \%)$ & \\
\hline \multicolumn{4}{|l|}{ Education level } \\
\hline $\begin{array}{l}\text { Elementary school } \\
\text { or lower }\end{array}$ & 75/1104 (6.8\%) & $25 / 368(6.8 \%)$ & \\
\hline Middle-high school & 678/1104 (61.4\%) & $226 / 368(61.4 \%)$ & \\
\hline College or higher & $351 / 1104$ (31.8\%) & $117 / 368(31.8 \%)$ & \\
\hline Depressive mood & & & $<0.001$ \\
\hline Yes & $108 / 1104(9.8 \%)$ & 131/367 (35.7\%) & \\
\hline No & 996/1104 (90.2\%) & $236 / 367$ (64.3\%) & \\
\hline Living alone & & & $<0.001$ \\
\hline Alone & $30 / 1104$ (2.7\%) & 66/367 (18.0\%) & \\
\hline Not alone & $1074 / 1104$ (97.3\%) & $301 / 367$ (82.0\%) & \\
\hline Sleep $<6 h$ & & & 0.775 \\
\hline$<6 \mathrm{~h}$ & 123/1104 (11.1\%) & 43/368 (11.7\%) & \\
\hline$\geqslant 6 \mathrm{~h}$ & $981 / 1104$ (88.9\%) & $325 / 368$ (88.3\%) & \\
\hline Marriage status & & & $<0.001$ \\
\hline Married & $853 / 1071$ (79.6\%) & $196 / 346(53.8 \%)$ & \\
\hline Single & 208/1071 (19.4\%) & $117 / 346(32.1 \%)$ & \\
\hline Divorced & 10/1071 (0.9\%) & $33 / 346(9.1 \%)$ & \\
\hline Stress level & & & 0.005 \\
\hline Very low & 130/1104 (11.8\%) & $46 / 367$ (12.5\%) & \\
\hline Low & 658/1104 (59.6\%) & $185 / 367(50.3 \%)$ & \\
\hline High & 266/1104 (24.1\%) & $107 / 367(29.1 \%)$ & \\
\hline Very high & $50 / 1104$ (4.5\%) & 29/367 (7.9\%) & \\
\hline Subjective body image & & & $<0.001$ \\
\hline Very skinny & $32 / 1104$ (2.9\%) & $32 / 366(8.7 \%)$ & \\
\hline Skinny & 128/1104 (11.6\%) & 70/366 (19.1\%) & \\
\hline Normal & $442 / 1104(40.0 \%)$ & $175 / 366(47.8 \%)$ & \\
\hline Fat & 423/1104 (38.3\%) & 73/366 (19.9\%) & \\
\hline Very fat & 79/1104 (7.1\%) & $16 / 366(4.4 \%)$ & \\
\hline Self-perceived health state & & & $<0.001$ \\
\hline Very good & $62 / 1104(5.6 \%)$ & $17 / 367(4.6 \%)$ & \\
\hline Good & $466 / 1104$ (42.2\%) & $79 / 367$ (21.5\%) & \\
\hline Normal & 408/1104 (37.0\%) & 170/367 (46.3\%) & \\
\hline Poor & $156 / 1104(14.1 \%)$ & $86 / 367$ (23.4\%) & \\
\hline Very poor & $12 / 1104$ (1.1\%) & $15 / 367(4.1 \%)$ & \\
\hline Abdominal obesity & & & 0.929 \\
\hline Yes & $319 / 1101$ (29.0\%) & $84 / 287$ (29.3\%) & \\
\hline No & 782/1101 (71.0\%) & 203/287 (70.7\%) & \\
\hline Monthly income $e^{a}$ & & & $<0.001$ \\
\hline$<1000000$ & $74 / 1085$ (6.8\%) & $78 / 254(30.7 \%)$ & \\
\hline $1000000-2000000$ & 210/1085 (19.4\%) & $67 / 254(26.4 \%)$ & \\
\hline $2000000-4000000$ & $461 / 1085$ (42.5\%) & $82 / 254(32.3 \%)$ & \\
\hline $4000000-6000000$ & 233/1085 (20.5\%) & $24 / 254(9.4 \%)$ & \\
\hline$>6000000$ & $107 / 1085(9.9 \%)$ & $3 / 254(1.2 \%)$ & \\
\hline Occupation & & & $<0.001$ \\
\hline Yes & $901 / 1104(81.6 \%)$ & $92 / 365(25.2 \%)$ & \\
\hline No & 203/1104 (18.4\%) & $273 / 365(74.8 \%)$ & \\
\hline Ownership of a house & & & $<0.001$ \\
\hline Yes & 756/1104 (68.5\%) & 195/368 (53.0\%) & \\
\hline No & $348 / 1104$ (31.5\%) & $173 / 368(47.0 \%)$ & \\
\hline $\begin{array}{l}\text { Poor familial property and } \\
\text { income }\end{array}$ & & & $<0.001$ \\
\hline Poor & $56 / 1104(5.1 \%)$ & $123 / 368$ (33.4\%) & \\
\hline Not poor & $1048 / 1104$ (94.9\%) & $245 / 368(66.6 \%)$ & \\
\hline
\end{tabular}

Abbreviations: $\mathrm{CG}$, control group; $\mathrm{SCl}$, spinal cord injury. ${ }^{a}$ Currency: Korean won. 
subjects with suicidal ideation, $17.3 \%(22 / 127)$ had attempted suicide in the SCI group and $8.7 \%(10 / 115)$ in the CG group $(P=0.048)$.

The factors significantly related to suicidal ideation in both the groups were female gender, high stress level in daily lives, poor selfperceived health state, alcohol dependence affecting normal life and depressive mood $(P<0.05)$. Low education level, $<6$ h of sleep a day, divorced marriage status, poor familial property and income were significantly related to suicidal ideation in only the SCI group $(P<0.05)$. In the SCI group, the proportion of persons with suicidal ideation did not significantly differ according to the completeness of injury (complete: $35.1 \%$, incomplete: $32.6 \%, P=0.623$ ) and the level of injury (tetraplegia: $38.7 \%$, paraplegia: $31.4 \%, P=0.156$ ). Cause of injury, passed time since injury and age at injury were not significantly related to suicidal ideation (data are not shown). In comparison, single marriage status, low abdominal circumference, low monthly income, unemployment and ownership of a house were significantly related to suicidal ideation in the CG group $(P<0.05$, Table 2). Age, living alone and subjective body image did not show significant relation with suicidal ideation (data are not shown).

Multiple logistic regression analysis for the whole sample (both the SCI and CG groups) revealed that depressive mood (odds ratio (OR) 6.284), SCI (OR 2.865), female gender (OR 2.285), very poor selfperceived health status (OR 4.855) and high stress levels (OR 3.741) were significant predictors of suicidal ideation (predictive percentage $86.8 \%, P<0.05$, Table 3). Depressive mood and female gender were significant risk factors in multiple logistic regression for the SCI group. The OR for depressive mood was 11.194 (confidence interval (CI) 6.507-19.255, $P<0.001)$ and the OR for female gender was 3.706 (CI $1.857-7.396, P<0.001$ ), with a predictive percentage of $77.5 \%$.

\section{DISCUSSION}

In this sample, $34.8 \%$ of individuals with chronic SCI reported having experienced suicidal ideation during the past year. This was $>3$ times than that of the CG, $10.4 \%$. The rate of suicidal attempt among the persons with suicidal ideation was significantly higher in the SCI group (17.3\%) compared with the CG (8.7\%), as was the overall rate of suicidal attempt. Results also indicated that SCI itself is a significant risk factor of suicidal ideation (OR 2.865). Depressive mood (OR 11.194) and female gender (OR 3.706) were also revealed as significant risk factors of suicidal ideation in the SCI group.

It is difficult to determine whether the rate of suicidal ideation is significantly higher in the Korean SCI population compared with other countries, particularly given that previous studies have primarily focused on suicide as the cause of death or SCI due to attempted suicide. $^{7,8}$ In comparison, the rate of suicidal ideation in this study was assessed by experience of the suicidal idea during the past year. Kishi et al. ${ }^{9}$ investigated on cross-sectional prevalence of suicidal ideation within 2 weeks before interview during subacute-to-chronic spinal rehabilitation, which is also hard to directly compare with this community-based sample. However, it seems definite that the rate of suicidal ideation is significantly higher than that of the age-, genderand education level-matched non-SCI population (CG), because we secured comparability by using the same question to investigate suicidal ideation for both the groups.

As described previously, the survey questions for SCI persons was developed as to match the annual national public survey for comparability. Twelve-month suicidal ideation and attempt are variables that were used for the KNHNES in Korea as well as National Comorbidity Survey Replication (NCS-R) in US. ${ }^{10}$ Variables such as depressive mood, subjective body image and stress in daily lives were also assessed by asking the prevalence within 1 year
Table 2 Chi-square tests for suicidal ideation in CG and SC

\begin{tabular}{|c|c|c|c|c|}
\hline & \multicolumn{2}{|l|}{$C G$} & \multicolumn{2}{|l|}{$S C l$} \\
\hline & \multirow{2}{*}{$\begin{array}{c}\text { Rate of suicidal } \\
\text { ideation }\end{array}$} & \multicolumn{3}{|c|}{ Rate of suicidal } \\
\hline & & P-value & ideation & P-value \\
\hline Gender (male/female) & & 0.007 & & $<0.001$ \\
\hline Male & $85 / 915$ (9.3\%) & \multicolumn{3}{|c|}{ 93/302 (30.8\%) } \\
\hline Female & $30 / 189$ (15.9\%) & \multicolumn{3}{|c|}{$34 / 63(54.0 \%)$} \\
\hline Education level & & 0.174 & & 0.008 \\
\hline Elementary school or lower & 11/75 (11.7\%) & \multicolumn{3}{|c|}{$15 / 25(60.0 \%)$} \\
\hline Middle-high school & 75/678 (11.1\%) & \multicolumn{3}{|c|}{$80 / 224(35.7 \%)$} \\
\hline College or higher & 29/351 (8.3\%) & \multicolumn{3}{|c|}{$32 / 116(27.6 \%)$} \\
\hline Sleep $<6 h$ & & 0.318 & & 0.040 \\
\hline$<6 h$ & 16/123 (13.0\%) & \multicolumn{3}{|c|}{$21 / 43(48.8 \%)$} \\
\hline$>6 \mathrm{~h}$ & 99/981 (10.1\%) & \multicolumn{3}{|c|}{$\begin{array}{l}106 / 322 \\
(32.9 \%)\end{array}$} \\
\hline Marriage status & & $<0.001$ & & 0.038 \\
\hline Married & $73 / 853(8.6 \%)$ & \multicolumn{3}{|c|}{ 66/196 (33.7\%) } \\
\hline Single & $37 / 208(17.8 \%)$ & \multicolumn{3}{|c|}{$35 / 116(30.2 \%)$} \\
\hline Divorced & $1 / 10(10.0 \%)$ & \multicolumn{3}{|c|}{$18 / 33(54.6 \%)$} \\
\hline Stress level & & $<0.001$ & & $<0.001$ \\
\hline Very low & $7 / 130(5.4 \%)$ & \multicolumn{3}{|c|}{$5 / 46(10.9 \%)$} \\
\hline Low & $43 / 658(6.5 \%)$ & \multicolumn{3}{|c|}{$51 / 182(28.0 \%)$} \\
\hline High & $50 / 266(18.8 \%)$ & \multicolumn{3}{|c|}{$56 / 107$ (52.3\%) } \\
\hline Very high & 15/50 (30.0\%) & \multicolumn{3}{|c|}{$15 / 29(51.7 \%)$} \\
\hline Self-perceived health state & & $<0.001$ & & 0.002 \\
\hline Very good & $5 / 62(8.1 \%)$ & & $3 / 17(17.7 \%)$ & \\
\hline Good & $35 / 466(7.5 \%)$ & & 21/77 (27.3\%) & \\
\hline Normal & $38 / 408$ (9.3\%) & & $53 / 170(31.2 \%)$ & \\
\hline Poor & $31 / 156$ (19.9\%) & & $39 / 85$ (45.9\%) & \\
\hline Very poor & $6 / 12(50.0 \%)$ & & $10 / 15(66.7 \%)$ & \\
\hline Abdominal obesity & & 0.042 & & 0.168 \\
\hline Obese & 24/139 (7.5\%) & & 26/84 (31.0\%) & \\
\hline Not obese & 91/781 (11.7\%) & & $80 / 202$ (39.6\%) & \\
\hline Monthly income ${ }^{a}$ & & 0.014 & & 0.156 \\
\hline$<1000000$ & $7 / 74(9.5 \%)$ & & $34 / 76$ (44.7\%) & \\
\hline $1000000-2000000$ & $38 / 210(18.1 \%)$ & & 24/66 (36.4\%) & \\
\hline $2000000-4000000$ & $44 / 461(9.5 \%)$ & & 25/84 (29.8\%) & \\
\hline $4000000-6000000$ & 20/233 (8.6\%) & & 4/24 (16.7\%) & \\
\hline$>6000000$ & $5 / 107(4.7 \%)$ & & $1 / 3(33.3 \%)$ & \\
\hline Occupation & & 0.001 & & 0.985 \\
\hline Yes & $81 / 901$ (9.0\%) & & 32/91 (35.2\%) & \\
\hline No & $34 / 203$ (16.8\%) & & 95/271 (35.1\%) & \\
\hline Alcohol dependence & & 0.029 & & 0.017 \\
\hline affecting normal life & & & & \\
\hline None & $59 / 640(9.2 \%)$ & & 54/163 (33.1\%) & \\
\hline Less than once a month & 22/159 (13.8\%) & & $17 / 40$ (42.5\%) & \\
\hline Once a month & $8 / 89(9.0 \%)$ & & 2/18 (11.1\%) & \\
\hline Once a week & $6 / 40(15.0 \%)$ & & $6 / 13(46.2 \%)$ & \\
\hline Almost every day & $2 / 4(50.0 \%)$ & & $3 / 3(100 \%)$ & \\
\hline Depressive mood & & $<0.001$ & & $<0.001$ \\
\hline Depressive & $42 / 108$ (38.9\%) & & $87 / 131(66.4 \%)$ & \\
\hline Not depressive & 73/996 (7.3\%) & & 40/234 (17.1\%) & \\
\hline Ownership of a house & & 0.007 & & 0.226 \\
\hline Yes & 66/756 (8.7\%) & & 62/194 (32.0\%) & \\
\hline No & 49/348 (14.1\%) & & 65/171 (38.0\%) & \\
\hline Poor familial property & & 0.061 & & 0.007 \\
\hline and income & & & & \\
\hline Yes & 10/56 (17.9\%) & & $54 / 122$ (44.3\%) & \\
\hline No & $\begin{array}{c}105 / 1048 \\
(10.0 \%)\end{array}$ & & 73/243 (30.0\%) & \\
\hline
\end{tabular}

Abbreviations: CG, control group; SCl, spinal cord injury. ${ }^{a}$ Currency: Korean won. 
Table 3 Multiple logistic regression model for all the subjects

\begin{tabular}{lccr}
\hline & Odds ratio & $95 \% \mathrm{Cl}$ & P-value \\
\hline Spinal cord injury & 2.865 & $2.027-4.049$ & $<0.001$ \\
Depressive mood & 6.284 & $4.359-9.058$ & $<0.001$ \\
Gender, female & 2.285 & $1.551-3.365$ & $<0.001$ \\
Self-perceived health status, very good & & & \\
$\quad$ Good & 1.152 & $0.488-2.270$ & 0.050 \\
$\quad$ Normal & 1.179 & $0.506-2.745$ & 0.703 \\
$\quad$ Poor & 1.533 & $0.637-3.687$ & 0.340 \\
$\quad$ Very poor & 4.855 & $1.379-17.096$ & 0.014 \\
Stress level, very low & & & \\
$\quad$ Low & & & $<0.001$ \\
$\quad$ High & 1.533 & $0.791-2.973$ & 0.206 \\
$\quad$ Very high & 3.741 & $1.892-7.397$ & $<0.001$ \\
\hline
\end{tabular}

Abbreviation: $\mathrm{Cl}$, confidence interval.

Prediction percentage: $86.8 \%$.

or rating psychological state of regular days, not on the day of survey. Although it is difficult to simply compare the rate of suicidal ideation with other studies, which have focused on lifetime prevalence or the rate at the time of investigation, ${ }^{4,9,11}$ analyzing the relationship between psychiatric or social parameters among the subjects, as done in this study, is still clinically meaningful.

Consistent with other studies, we found that depressive mood is significantly related with suicidal ideation in both the SCI and CG groups. ${ }^{12}$ The results also suggest that SCI has an additive effect on suicidal ideation in coexistence with depressive mood. Although associated factors such as low education level, marital status, low socioeconomic state and sleep deprivation were not selected as risk factors in the regression analysis, we can infer that these factors could have acted as co-factors with depressive mood. Indeed, several studies have demonstrated that low education level, unemployment and lower socioeconomic status are significant risk factors of depression in SCI patients. ${ }^{13-15}$

This study also revealed that female gender, low education level, marital status (single in the CG and divorced state in the SCI group), high stress level in daily lives, poor self-perceived health state and alcohol dependence affecting normal life were significantly related with suicidal ideation in both the CG and SCI groups. In the CG, low monthly income, unemployment and no possession of own house were all significantly related with suicidal ideation, but in the SCI group, only receiving partial financial support from the government, which means that total familial income level is lower than the minimum cost of living, was significantly related. It appears that in general population individual financial or economic problems contribute to the development of suicidal ideation, whereaas in the SCI population, the overall familial financial state affect the suicidal thought. Many other studies also support that unemployment, low job compatibility, unmarried state, absence of stress reduction technique, female gender, low education level, childhood trauma, previous mental disorder, poor self-perceived health state, financial strain and poor social support are risk factors of suicide in general population. ${ }^{12,16-18}$ But Almeida et al. ${ }^{18}$ insisted that male gender and higher education level were independently associated with suicidal ideation, which is opposite to our study.

In all the analyses, the level and motor completeness of injury did not have significant association with suicidal ideation. However, the level of injury was significantly related with depressive mood
$(P=0.007)$. It appears that the characteristics of the injury itself are not important in arousal of suicidal behaviors. This result concurs with the study of Hartkopp et al., ${ }^{3}$ which reported no difference in suicide rate regarding functional group or level of the lesion.

There are several limitations in this study. First, the data were based on self-report and not on clinician-based ratings. In particular, medical records were not available for subjects, hence the selfreported information on level or motor completeness of the injury may have been inaccurate in some cases. Second, the questionnaire was purposely designed for the study, hence some categories such as suicidal ideation and depressive mood were not based on standard assessment tools. Furthermore, the survey item used to assess depressive mood (for example, having feelings of sadness or despair affecting their daily lives in consecutive $\geqslant 2$ weeks during the past year) comprised just one of the main criteria for major depressive disorder in the Diagnostic and Statistical Manual of Mental Disorders, Fourth Edition. ${ }^{19}$ As such, depression measured by a standardized tool and depressive mood as measured in this study may each have a different influence on suicidal ideation. Third, the subjects of this study may have had better psychosocial adjustment than the general Korean SCI population due to sampling bias, given that all SCI subjects had been recruited from an internet database.

\section{CONCLUSION}

Suicidal ideation was more frequent among Korean persons with SCI compared with a CG, and closely related to psychosocial adjustment. Moreover, suicidal ideation was not significantly related to the level or completeness of injury. The findings suggest that screening for suicidal ideation and psychosocial interventions regardless of injury severity would be necessary to prevent suicide in persons with SCI. Further research on suicidality in the SCI population, utilizing standardized medical assessment and examining the efficacy of preventive interventions, will help to properly manage psychosocial problems.

\section{DATA ARCHIVING}

There were no data to deposit.

\section{CONFLICT OF INTEREST}

The authors declare no conflict of interest.

1 Mackenzie TB, Popkin MK. Suicide in the medical patient. Int J Psychiatr Med 1987; 17: 3-22.

2 DeVivo MJ, Black KJ, Richards JS, Stover SL. Suicide following spinal cord injury. Paraplegia 1991; 29: 620-627.

3 Hartkopp A, Bronnum-Hansen H, Seidenschnur AM, Biering-Sorensen F. Suicide in a spinal cord injured population: its relation to functional status. Arch Phys Med Rehab 1998; 79: 1356-1361.

4 Kishi Y, Robinson RG, Kosier JT. Suicidal ideation among patients with acute lifethreatening physical illness: patients with stroke, traumatic brain injury, myocardial infarction, and spinal cord injury. Psychosomatics 2001; 42: 382-390.

5 Kim SW, Yoon JS. Suicide, an urgent health issue in Korea. J Korean Med Sci 2013; 28: 345-347.

6 Lee S, Park HS, Kim SM, Kwon HS, Kim DY, Kim DJ et al. Cut-off points of waist circumference for defining abdominal obesity in the Korean population. Korean J Obes 2006; 15: 1-9.

7 Lombardi G, Mondaini N, lazzetta P, Macchiarella A, Del Popolo G. Sexuality in patients with spinal cord injuries due to attempted suicide. Spinal Cord 2008; 46: 53-57.

8 Thietje R, Pouw MH, Schulz AP, Kienast B, Hirschfeld S. Mortality in patients with traumatic spinal cord injury: descriptive analysis of 62 deceased subjects. J Spinal Cord Med 2011; 34: 482-487.

9 Kishi Y, Robinson RG, Kosier JT. Suicidal ideation among patients during the rehabilitation period after life-threatening physical illness. J Nerv Ment Dis 2001; 189: 623-628. 
10 Bodenlos JS, Lemon SC, Schneider KL, August MA, Pagoto SL. Associations of mood and anxiety disorders with obesity: comparisons by ethnicity. J Psychosom Res 2011; 71: 319-324.

11 Pinkerton AC, Griffin ML. Rehabilitation outcomes in females with spinal cord injury: a follow-up study. Paraplegia 1983; 21: 166-175.

12 Miret M, Ayuso-Mateos JL, Sanchez-Moreno J, Vieta E. Depressive disorders and suicide: epidemiology, risk factors, and burden. Neurosci Biobehav Rev 2013; S0149-7634: 00009-2.

13 Bombardier CH, Fann JR, Tate DG, Richards JS, Wilson CS, Warren AM et al. An exploration of modifiable risk factors for depression after spinal cord injury: which factors should we target? Arch Phys Med Rehab 2012; 93: 775-781.

14 Arango-Lasprilla JC, Ketchum JM, Starkweather A, Nicholls E, Wilk AR. Factors predicting depression among persons with spinal cord injury 1 to 5 years post injury. NeuroRehabilitation 2011; 29: 9-21.
15 Krause JS, Kemp B, Coker J. Depression after spinal cord injury: relation to gender, ethnicity, aging, and socioeconomic indicators. Arch Phys Med Rehab 2000; 81: 1099-1109.

16 Ten Have M, van Dorsselaer S, de Graaf R. Prevalence and risk factors for first onset of suicidal behaviors in the Netherlands Mental Health Survey and Incidence Study-2. J Affect Disorders 2013; 147: 205-211.

17 Sugawara N, Yasui-Furukori N, Sasaki G, Tanaka O, Umeda T, Takahashi I et al. Gender differences in factors associated with suicidal ideation and depressive symptoms among middle-aged workers in Japan. Ind Health 2013; 51: 202-213.

18 Almeida OP, Draper B, Snowdon J, Lautenschlager NT, Pirkis J, Byrne G et al. Factors associated with suicidal thoughts in a large community study of older adults. $\mathrm{Br} J$ Psychiatr 2012; 201: 466-472.

19 American Psychiatric Association. Diagnostic and Statistical Manual of Mental Disorders, 4th Edn. American Psychiatric Association: Washington, DC, USA, 1994. 